\title{
Development of a hexagonal, mesh-based distribution method for community health centres
}

\author{
Yinan Zhou, ${ }^{1}$ Ge Bai, ${ }^{2} \mathrm{Li} \mathrm{Luo}^{2}$ \\ ${ }^{1}$ School of Public Health; ${ }^{2}$ Research Institute of Health Development Strategies \& Collaborative Innovation \\ Center of Social Risks Governance in Health, Fudan University, Shanghai, China
}

\begin{abstract}
Community health centres (CHCs) are the health gatekeepers of the local population. Location and numbers of the CHCs affect fairness and effectiveness with regard to access to primary health care. In the past, the distribution of the CHCs was solely empiric-based. The goal of this study was to devise a method for $\mathrm{CHC}$ distribution based on the principle of improving equity as well as ensuring efficiency. We tested the effectiveness and operability of the method through a process of revision and simulation using Guangdong Province, China as sample district. A methodology based on literature review and expert consultation was repeatedly applied until an ideal result had been reached. A hexagonal, mesh-based method was developed and used to find a solution where the CHCs could be distributed where their location would be the most needed and total number suitable. Testing the effectiveness of the proposed plan, we
\end{abstract}

Correspondence: Li Luo, Research Institute of Health Development Strategies \& Collaborative Innovation Center of Social Risks Governance in Health, Fudan University, Shanghai, China.

E-mail: liluo@fudan.edu.cn

Acknowledgements: the authors would like to thank the participants for their contribution to this study.

Key words: Access to health care; GIS; Primary health care; China.

Contributions: LL: concept design, data acquisition, approval of the final version of the paper. YZ: data collection, paper writing; GB: article revision.

Conflict of interest: the authors declare no potential conflict of interest.

Funding: the Research Foundation from Ministry of Education of China (Grant No. 15JZD029). National Natural Science Foundation of China (Grant No. 71503053).

Received for publication: 9 November 2017.

Revision received: 25 July 2018

Accepted for publication: 25 July 2018.

(C) Copyright Y. Zhou et al., 2018

Licensee PAGEPress, Italy

Geospatial Health 2018; 13:648

doi:10.4081/gh.2018.648

This article is distributed under the terms of the Creative Commons Attribution Noncommercial License (CC BY-NC 4.0) which permits any noncommercial use, distribution, and reproduction in any medium, provided the original author(s) and source are credited. found the proportion of area covered to be $52.8 \%$ and the proportion of the population covered $80.7 \%$, which is $15.4 \%$ and $14.7 \%$, respectively, better than before. It was concluded that the hexagonal mesh-based, distribution method can effectively define the location as well as the number or required CHCs, not only improving the accessibility for residents to primary health care services but also maximizing cost-effectiveness. Management of the city by grid is a new idea in urban management, which improves rationality of planning and also may be applied for many different purposes in addition to CHC localization.

\section{Introduction}

Community Health Centres (CHCs) represent the most prevalent community-level form of healthcare connection available to the general public. They are the first point of contact between the family/community and the national health system bringing health care as close as possible to where people live and work. Although the World Health Organization (WHO) put forward the slogan Health for All by 2000 in the Declaration of Alma-Ata 1978, (WHO, 1978), seven out of 10 CHC patients in North America still live in poverty, and well over half are members of minority groups (Adashi et al., 2010). These authors also point out that the $\mathrm{CHC}$ is often the sole health care provider available to these patients. CHC planning is therefore critical to achieve full health coverage for all people. However, there are relatively few studies on $\mathrm{CHCs}$ distribution. In the past, planning was often done empirically or by using administrative divisions. Access to primary health care varies from one area to another because health services are affected by the location (supply) of the $\mathrm{CHC}$ and patient residence location (demand), and neither health professionals nor populations are uniformly distributed.

In order to achieve the goal of primary health care for everyone, it is important to establish the proper location and capacity of available CHCs. Recently, geographical information systems (GIS) and related spatial techniques have been used for analyzing the relationship between health outcomes and accessibility, as well as better health services (Moore and Carpenter 1999; Bell et al., 2006; Sasaki et al., 2008; Lee, 2013). In the context of current and future public health needs, it is possible to analyze the role of supporting public health programmes, such as maximizing resource efficiency (Luo and Wang, 2003; Sasaki et al., 2010). To identify areas where it would be useful to locate $\mathrm{CHCs}$, researchers aggregate the city into accessibility zones, and planners use the zones to decide whether currently available health facilities cover the entire city or not. This method is useful for the evaluation (Luo and Wang, 2003), as the areas uncovered by CHCs are clearly visualized. However, the approach leads only to the identification of 
approximate areas that should benefit by an additional CHCs, so the question of exact numbers and locations of CHCs to be added without duplication or omission remains partly unsolved. In addition, the cost-effectiveness of adding new CHCs is low in areas with low populations, since maintenance of operations requires a certain minimum number of people.

The goal of this study, based on the principle of improving equity as well as ensuring efficiency, was to develop a method of assessing which areas require new $\mathrm{CHC}$ and if so where to localize them. Cost-effectiveness and accessibility should be maximized with a minimum increase of the number of $\mathrm{CHCs}$.

\section{Materials and Methods}

Increasing equity does not entail sacrificing efficiency, e.g., if the number of CHCs were significantly increased, equity would certainly be improved, but cost-effectiveness might decrease. We propose instead to maximize accessibility to primary health care for all residents in a way that also makes sense financially.

\section{Literature review}

In order to solve these problems encountered with regard to $\mathrm{CHC}$ localization, we conducted a literature review. Taking accessibility/spatial accessibility as the key word, we searched China National Knowledge Infrastructure, the PubMed, the Web of Knowledge databases. After screening, a total of 170 papers were obtained. 64 articles related to the spatial accessibility evaluation method, 11 to process analysis, 39 to index construction, 56 to topics of visual expression (including 6 articles referring to spatial location determination), 19 to road network distance calculation, 23 to Population spatial distribution simulation and 8 to the grid data model. This literature search contributed considerably in providing both a theoretical and a methodological basis for this study.

\section{Expert consultations}

We also engaged in consultation with experts. These experts included individuals in charge of $\mathrm{CHCs}$, health bureau staff, school teachers and experts in health planning. After comparing the difference between theoretical results and actual experience, we adjusted and perfected our method recursively. Finally, we established a method for estimating the best distribution of CHCs based on a hexagonal mesh. The roadmap for establishment of the methodology is shown schematically in Figure 1.

\section{Data simulation}

In order to test the operability and validity of the method, we selected a sample district in Guangdong Province, China as an example. We have previously applied this method to make policy recommendations for the rational distribution of community health service stations for other areas, such as Songjiang and Hongkou districts in Shanghai and also to Jinjiang District of Fujian Province, which indicated that this method could be generalized. Our aim now was to demonstrate the usefulness of the approach in one specific district in Guangdong Province. Table 1 displays the list at the county level of data and sources corresponding to our research.

\section{Software}

Primary data organization and analysis were performed using Microsoft Excel, 2013.

Mapping and calculation of spatial analysis were performed using ArcGIS v10.1 by ESRI (Redlands, CA, USA).

\section{The community health centres distribution plan}

In order to achieve the goal of full coverage, the number of CHCs in underserved areas would need to increase. Our strategy was to divide the region using a grid, counting the number of CHCs and the number of people in each cell of the area, then determining the location and number of $\mathrm{CHCs}$ needed for full coverage.

\section{Theoretical fundament}

A CHC service area can be described as an area with the $\mathrm{CHC}$ near the centre. Its shape would ideally be a circle, but geographical areas cannot be seamlessly apportioned in that way (Figure 2), so we decided to introduce the hexagonal form. The hexagon approximates the circular ideal of a service coverage area, while at the same time satisfying both the demands of comprehensiveness and the absence of overlap, thus avoiding duplication or omission of any services (Figure 2). Importantly, hexagons, like squares and like-sided triangles, can easily be laid out side by side without running into the tiling problem where angels do not fit.

The radius of each hexagon determines the maximum walking distance to a CHC from people's homes. According to WHO's requirement for developing countries, the range of a $\mathrm{CHC}$ service area should be within 15 minutes on foot. According to expert consultation, we set the average walking time as $100 \mathrm{~m}$ per minute, making $1,500 \mathrm{~m}$ the chosen radius of each hexagon as seen in Figure 2.

With at least one $\mathrm{CHC}$ in each hexagon, full coverage in a given area can be ensured. However, the number of $\mathrm{CHCs}$ also depends on the population. If its density would be very high in an area, one $\mathrm{CHC}$ might not be capable to fully meet the needs of the residents there. Conversely, if the population base would be very low, the cost-effectiveness of adding $\mathrm{CHCs}$ would also be low. Thus, we needed to develop a criterion for adjusting CHC numbers based on geography as well as population. According to the existing situation and expert consultation, we decided that the greatest number of people to be served by one $\mathrm{CHC}$ should be approximately 10,000 , while the lowest should be somewhere around

Table 1. Overview of data acquired.

\begin{tabular}{lccc} 
Category & Type of information & Level & Source \\
Population data & Distribution of people in 267 villages & County & Health Bureau \\
Geographic data & Digital maps & County & Bureau for urban planning and design \\
\hline & Digital map of road network & County & Bureau for urban planning and design \\
CHC & CHC distribution & County & December2015 \\
\hline
\end{tabular}

CHC, community health centres. 
5,000. For areas in which the population was low or varied between 5,000 and 10,000, the hexagons were merged until the population reached 10,000. Additionally, areas with an insufficient number of $\mathrm{CHCs}$ in relation to the population would receive one $\mathrm{CHC}$ for each additional 10,000 population, while no $\mathrm{CHC}$ was added to areas with less than 5,000 people. The criteria for adding CHCs are displayed in Table 2.

\section{Empirical approach}

We conducted a simulation in the sample district in Guangdong Province. Based on the 15-minute access requirement, the area was divided by means of GIS software into $392 \mathrm{CHC}$ service areas, each represented by a hexagon (Figure 3).

To determine the appropriate number and location of CHCs, we first summarized the number of $\mathrm{CHCs}$ in each hexagon and then located the areas without CHCs. There were 249 hexagons without CHCs (Table 3, Figure 4). These non-CHC areas, amounting to $63.5 \%$ of the total number, were considered priority localizations for additional CHCs.

\section{Evaluation}

Accessibility can be implemented based on spatial and non-

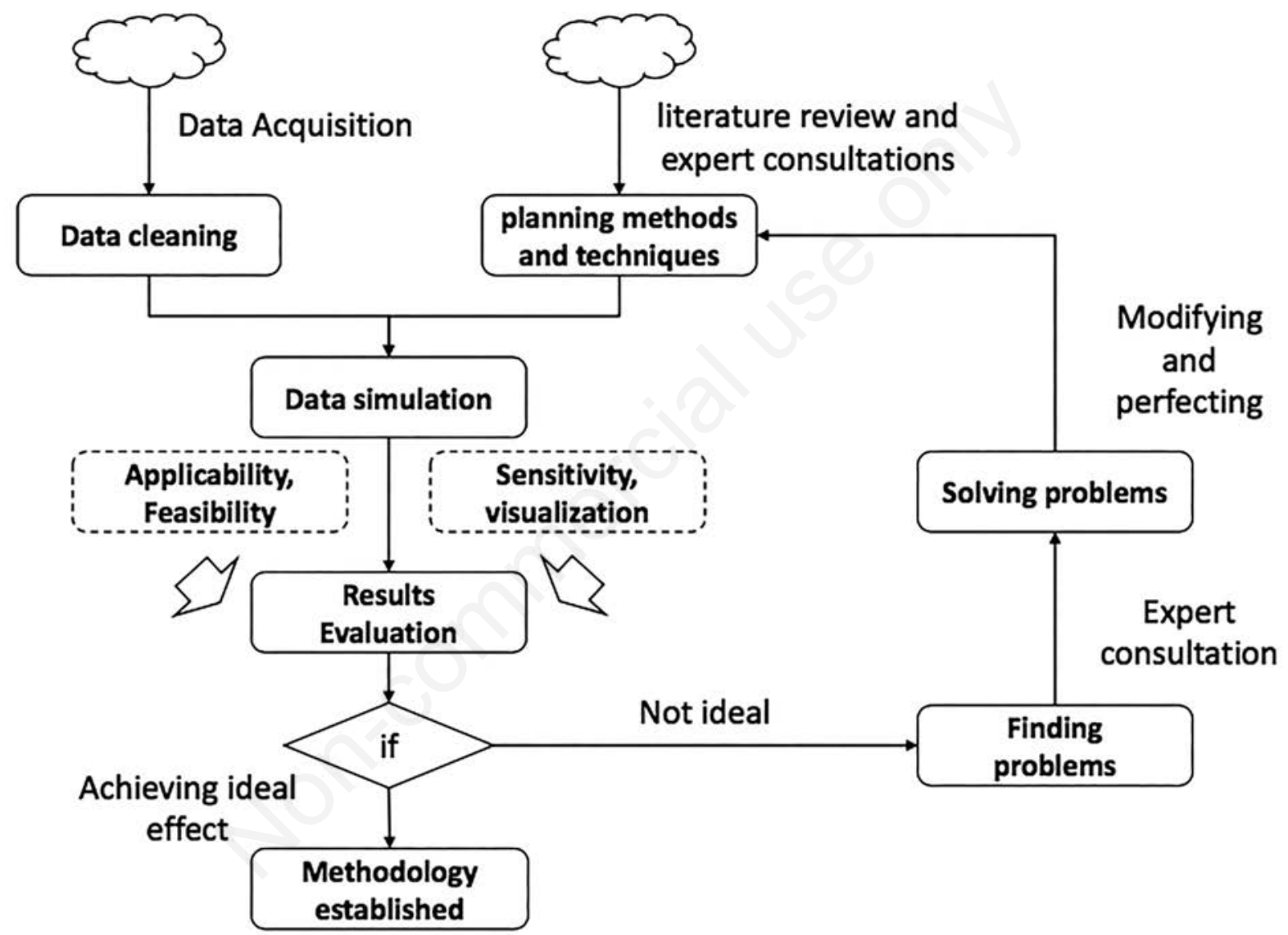

Figure 1. Technical roadmap for establishing the methodology.

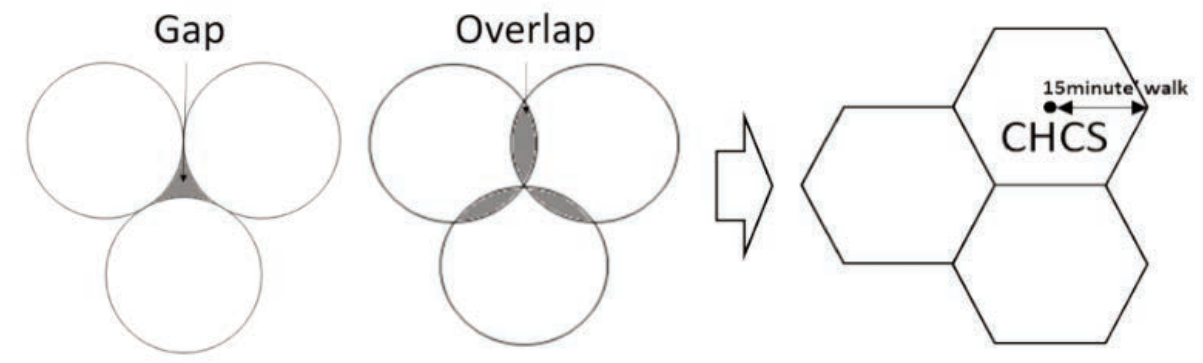

Figure 2. Graphical representation of service areas and the range of each hexagonal grid. CHC, community health centres. 
spatial factors (Murad, 2007). According to the purpose of this study, we only considered the spatial accessibility, i.e. the provider-to-population ratio. The accessibility of health services should be an important indicator to evaluate the methodological effectiveness.

The actual service area was used to measure the range of CHCs providing primary health care to residents. This area has the $\mathrm{CHC}$ near the centre, and 15-minute walk's distance is used as the distance limit. To reflect the effectiveness of $\mathrm{CHC}$ distribution, we used two indices when estimating the CHC service area: the proportion of area coverage, and the proportion of population coverage (Zhou et al., 2016).

\section{Results}

We summed the number of people in each hexagon, and calculated the number of CHCs needed. Together, there were 117,751 people $(42.0 \%$ of the total population) in the non-CHC areas. Out of the 249 non-CHC hexagons, 170 hexagons had populations lower than 5,000, 57 hexagons had populations between 5,000 and 10,000 , while 27 hexagons had populations of between 10,000 and 20,000 . Our proposal entails not adding any CHCs in lowest populated areas as it would not be cost-effective, while adding 22
Table 2. Criteria for community health centre (CHC) adjustment.

\begin{tabular}{lcc} 
Category & Population & Proposal \\
Areas without & $\leq 5000$ & No CHC addition \\
CHCs & $5,000-10,000$ & Add CHC after \\
& $10,000-20,000$ & area combination \\
& & Add one \\
& or two CHC(s) \\
Areas with CHCs & $\geq 10,000$ & Add one CHC for \\
& & each additional \\
& 10,000 population \\
\hline
\end{tabular}

Table 3. CHC distribution.

\begin{tabular}{cc} 
Number of CHCs in each hexagon & Number of hexagons \\
0 & 249 \\
1 & 107 \\
\hline 2 & 27 \\
3 & 6 \\
\hline 4 & 1 \\
5 & 1 \\
\hline 6 & 1 \\
Total & 392 \\
\hline
\end{tabular}

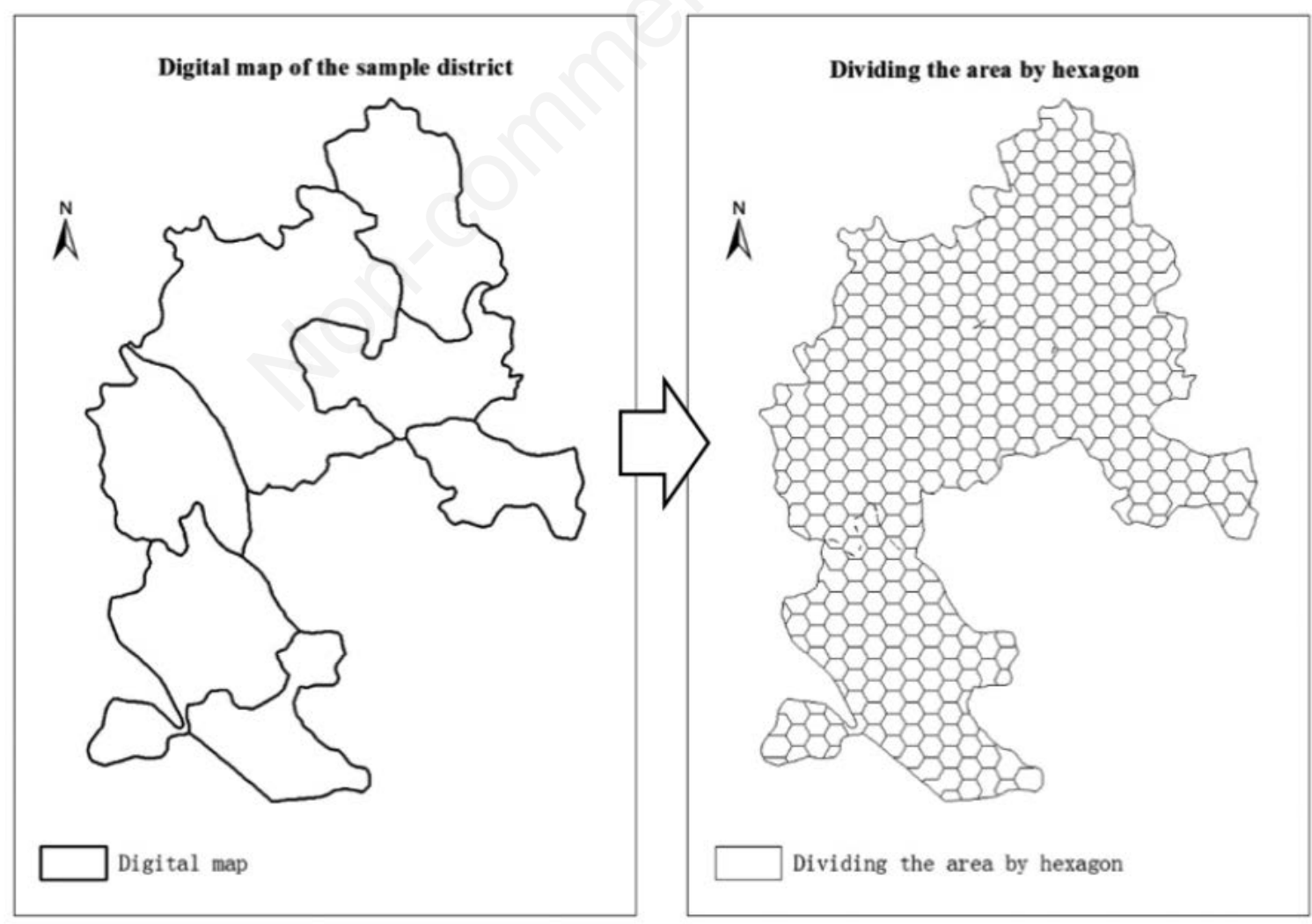

Figure 3. The sample area in Guangdong and its division into hexagons. 
CHCs to the 57 moderately populated hexagons after merging some of them. For the areas covered by the 27 hexagons with high populations, $27 \mathrm{CHCs}$ should be added. (Table 4, Figure 4).

Out of the hexagons that already had a CHC, one had between 20,000 and 30,000 people, a situation requiring an estimated two more $\mathrm{CHCs}$ according to our calculations. Eight hexagons with populations between 10,000 and 20,000, would each need an additional $\mathrm{CHC}$, i.e. eight $\mathrm{CHCs}$ in all (Table 4, Figure 4).

After planning, the proportion of area coverage of CHCs service area was $52.8 \%$, which is $15.4 \%$ higher than before. The ratio of population coverage was $80.7 \%$, which is $14.7 \%$ higher than before (Table 5, Figure 5).

\section{Discussion and Conclusions}

A hexagonal, mesh-based distribution method can effectively define the location and number of needed $\mathrm{CHCs}$ with maximum cost-effectiveness. Maximum accessibility is achieved, while a minimum of CHCs need to be added. The hexagonal grid division avoids all duplication or omission of coverage, and all people in each hexagon are guaranteed health support. This method thus achieves the planning objectives to improve equity as well as to ensure efficiency, and CHCs are added to the areas most in need. In consequence, not only will geographical accessibility to primary

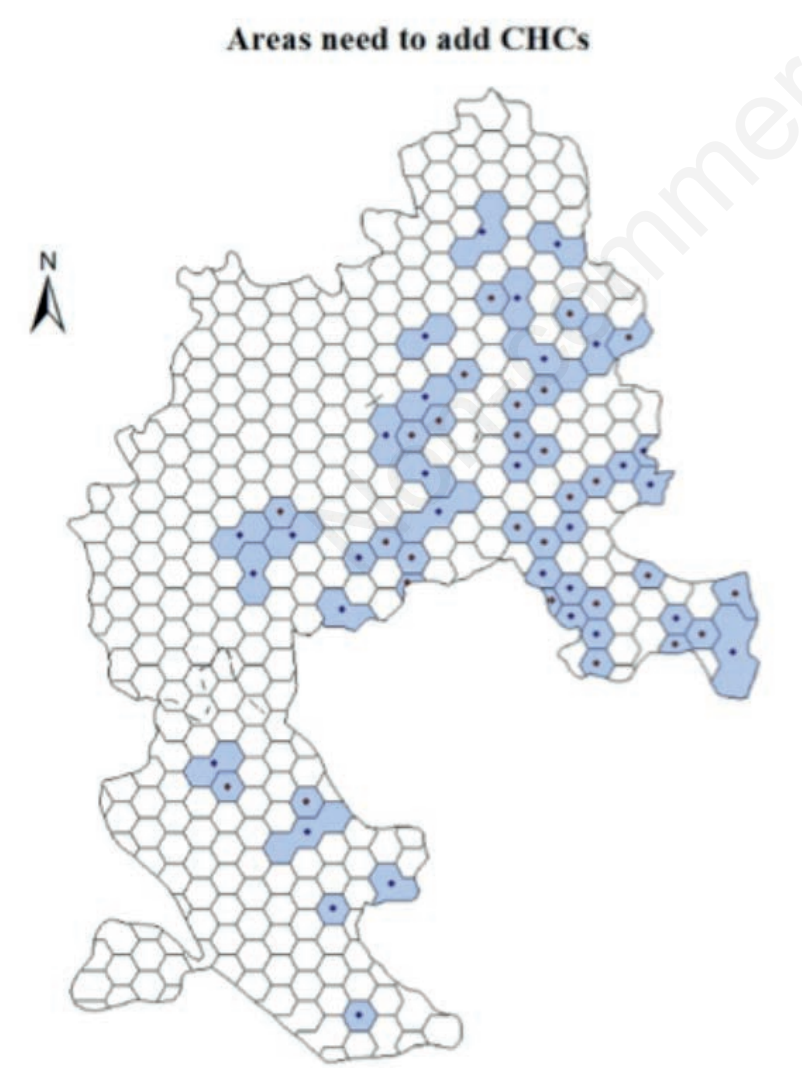

Areas need to add CHCs

Figure 4. Areas where community health centres (CHC) should be added. A dot represents one CHC. health care services be strengthened, but by taking the variation of population in each area, crowding, as well as under utilization, are avoided at each $\mathrm{CHC}$, which is the key factor to maintain operation.

Table 4. Current population distribution and estimated $\mathrm{CHC}$ requirement.

\begin{tabular}{lccc} 
Category & $\begin{array}{c}\text { Population } \\
\text { Areas without }\end{array}$ & $\begin{array}{c}\text { Number of } \\
\text { hexagons }\end{array}$ & $\begin{array}{c}\text { Additional CHCs } \\
\text { required }\end{array}$ \\
CHCs & $5000-10000$ & 170 & 0 \\
& $10000-20000$ & 52 & 22 \\
Areas with & $10000-20000$ & 8 & 27 \\
CHCs & $20000-30000$ & 1 & 8 \\
\hline
\end{tabular}

Table 5. Evaluation of the distribution plan effect.

\begin{tabular}{lccc}
\hline Category & $\begin{array}{c}\text { Before } \\
\text { planning }\end{array}$ & $\begin{array}{c}\text { After } \\
\text { planning }\end{array}$ & Increase \\
$\begin{array}{l}\text { Proportion of area } \\
\text { coverage }\end{array}$ & $43.5 \%$ & $52.8 \%$ & $9.3 \%$ \\
$\begin{array}{l}\text { Proportion of population } \\
\text { coverage }\end{array}$ & $66.0 \%$ & $80.7 \%$ & $14.7 \%$ \\
\hline
\end{tabular}

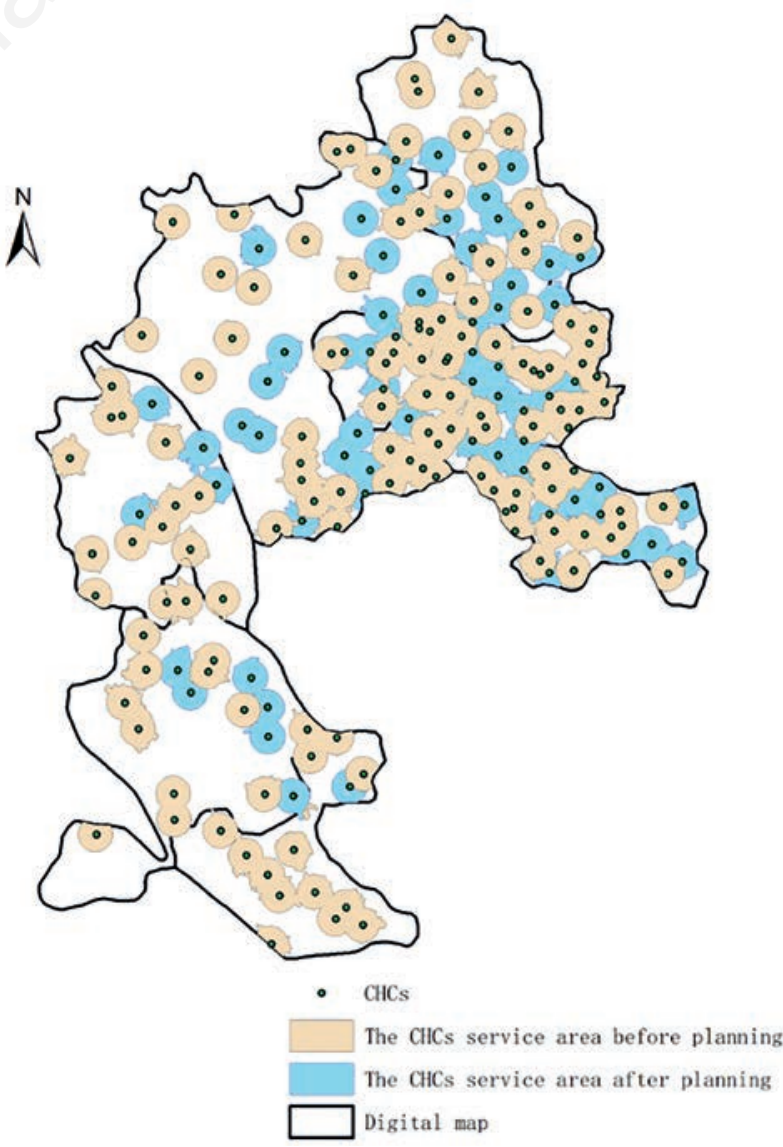

Figure 5. Effect of the distribution plan. 
At present, urban management by grid has become a new idea in urban management. How to provide citizens with more convenient public management and public services are among the most prominent problems faced by city governments in modern cities. Establishment of new methods of urban management is urgent.

Visual expression makes the method easily understood and accepted. Indeed, mapping is an excellent means of communicating a message clearly even to those who are not necessarily familiar with the methodology. Visualization of CHCs allows abstract concepts to be translated into intuitive, understandable graphics that convey scientific research conclusions to decision makers in a concise and understandable way, enabling them to quickly understand and adopt.

The reliability and validity are the criteria used to evaluate the quality of the method. Reliability can be guaranteed by consistency and stability of the method. The consistence and stability of basic data are guaranteed by the standard type, formulation and sources, and the consistence and stability of calculation process and result are guaranteed by the standard methodology to deal with data and visualize the results. The validity of the method is ensured by the actual increase in accessibility of residents to primary health care shown here and also judged by experts.

The fact that location of the grids may affect the result slightly is a limitation of the work since moving the grids would change the number of population and the number of CHCs counted. However, we have done relevant studies to examine the effect of different grid positions on the final results, which shows that it only has a marginal effect. In addition, no matter which grid the CHCs and population are in, the purpose of our study is to find out the areas where supply and demand do not match.

Although we only considered spatial accessibility, we understand that the health needs are also related to other factors, e.g., the proportion of elderly population, the level of economic development and so on. As it will affect the number of CHCs needed, we plan to improve our methodology by also take these factors into account in future studies.

This paper is a methodological study with a focus on health care, but urban grid division applied in this paper can be generalized. Thus, the approach proposed can not only be used for the localization of $\mathrm{CHCs}$, but also for other fixed structures in the built environment, e.g., pharmacies, convenience stores, etc. It would only be needed to define the size of each grid based on the particular purpose chosen.

\section{References}

Adashi EY, Geiger HJ, Fine MD, 2010. Health care reform and primary care - the growing importance of the community health center. N Engl J Med 362:2047-50.

Bell BS, Hoskins RE, Pickle LW, Wartenberg D, 2006. Current practices in spatial analysis of cancer data: mapping health statistics to inform policymakers and the public. Int $\mathrm{J}$ Health Geogr 5:49.

Lee KS, 2013. Disparity in the spatial distribution of clinics within a metropolitan city. Geospat Health 7:199-207.

Luo W, Wang F, 2003. Measures of spatial accessibility to health care in a GIS environment: synthesis and a case study in the Chicago region. Environ Plan B: Urban Analytics City Sci 30:865-84.

Moore DA, Carpenter TE, 1999. Spatial analytical methods and geographic information systems: use in health research and epidemiology. Epidemiol Rev 21:143-61.

Murad A, 2007. A GIS application for modeling accessibility to health care centers in Jeddah, Saudi Arabia. In: Lai PC, Mak ASH, eds. GIS for Health and the Environment. Lecture Notes in Geoinformation and Cartography. Berlin, Heidelberg: Springer. pp 57-70.

Sasaki S, Comber AJ, Suzuki H, Brunsdon C, 2010. Using genetic algorithms to optimise current and future health planning-the example of ambulance locations. Int J Health Geogr 9:4.

Sasaki S, Suzuki H, Igarashi K, Tambatamba B, Mulenga B, 2008. Spatial analysis of risk factor of cholera outbreak for 20032004 in a peri-urban area of Lusaka, Zambia. Am J Trop Med Hyg 79:414-21.

World Health Organization, 1978. Report of the International Conference on Primary Health Care. Alma-Ata, USSR, 6-12 September 1978. 79 pp.

Zhou YN, Bai G, Shen H, 2016. Evaluation method and empirical research on the distribution planning effects of community health centers. Chin Primary Health Care 30:15-6. 